UT-861

\title{
A configuration of 11-dimensional curved superspace as backgrounds for supermembrane
}

\author{
Shibusa Yuuichirou \\ Department of Physics, University of Tokyo \\ Hongo 7-3-1, Bunkyo-ku, Tokyo 113-0033, Japan
}

\begin{abstract}
We calculate all of vielbein superfields up to second order in anticommuting coordinates in terms of the component fields of 11-dimensional on-shell supergravity by using 'Gauge completion'. This configuration of superspace holds the $\kappa$-symmetry for supermembrane Lagrangian and represents 11-dimensional on-shell supergravity.
\end{abstract}




\section{Introduction}

Some years ago, T. Banks, W. Fischler, S. H. Shenker and L. Susskind (BFSS) proposed that Matrix theory gives a complete description of light-front M-theory [1]. It had been proposed as a theory of D0-branes by E. Witten [2].

Up to now, it has become clear that Matrix model encodes a remarkable amount of the structure of M-theory and 11-dimensional supergravity(for reviews, see [3]). The interaction between gravitons in Matrix theory has been shown to agree with supergravity to some extent [四].

However, this theory is constructed on flat spacetime, therefore Matrix theory on curved backgrounds is required. For single D0-branes, the theory on curved backgrounds is expected to be described by Born-Infeld action [5]. For multi-particle system of D0branes, namely Matrix model, the theory on curved backgrounds is as yet unknown. There are many trials to this problem. For example, starting from flat Matrix theory, backgrounds are produced by many D0-branes [6]. The other idea is that it is expected as supermembrane on curved backgrounds [7]. In this paper we adopt the later idea.

The theory of supermembrane is described as nonlinear sigma model [8]. Supermembrane consistently couples to 11-dimensional superspace backgrounds that satisfy a number of constraints which are equivalent to 11-dimensional on-shell supergravity [9]. After light cone gauge fixing and $\kappa$-symmetry gauge fixing, supermembrane theory on flat backgrounds is equivalent to a quantum-mechanical model with supersymmetric $\mathrm{U}(\mathrm{N})$ gauge symmetry in the large $\mathrm{N}$ limit by use of matrix regularization [10]. It has a continuous mass spectrum and instability [11], therefore it is expected that supermembrane matrix theory describes second quantization of D0-branes [12]. From the beginning of sigma model, it couples to general backgrounds, therefore it is expected that sigma model on curved backgrounds is a candidate of Matrix theory on curved backgrounds. Actually curved backgrounds for supermembrane were investigated [7]. In this reference, they calculated part of vielbein superfields and all of 3-form superfields up to second order in anticommuting coordinates.

On the other hand, there are two more aspects of importance of searching for the structure of 11-dimensional superspace. One is getting much knowledge of many low dimensional supergravity theories which can be obtained by dimensional reduction from 11-dimensional supergravity. The other aspect is getting the Lagrangian of superparticle coupled to 11-dimensional curved backgrounds. It is not Matrix theory, but very similar to Matrix theory 13 .

However the structure of 11-dimensional superspace was not yet well-known. Thus we investigate the more higher components of 11-dimensional superspace. 
In this paper, we compute all of vielbein superfields up to second order in anticommuting coordinates in terms of the component fields of 11-dimensional on-shell supergravity by using 'Gauge completion'. This configuration of superspace holds the $\kappa$-symmetry for supermembrane Lagrangian and represents 11-dimensional on-shell supergravity.

The paper is organized as follows. In section 2, we explain our notations of the 11-dimensional supergravity and obtain the full algebra of transformations in component formalism. In section 3, we review the supermembrane theory and the condition of $\kappa$-symmetry and explain our notations of the superspace geometry and obtain the full algebra of transformations in superspace and solve Bianchi identity in our obtained constraints. In section 4, we explain 'gauge completion' and compute part of the superfields. Other notations and conventions used throughout this paper are summarized in Appendix.

\section{11-dimensional supergravity}

Supergravity in 11-dimensional spacetime is based on 'elfbein' field $e_{m}^{a}$, a Majorana gravitino field $\psi_{m}^{\alpha}$ and third rank antisymmetric gauge field $C_{k l m}$. Its Lagrangian can be written as follows [14][7].

$$
\begin{aligned}
L= & -\frac{1}{2} e R-2 e \bar{\psi}_{m} \Gamma^{m n l} D_{n}\left(\frac{1}{2}(\omega+\hat{\omega})\right) \psi_{l}-\frac{1}{96} e F^{2} \\
& +\frac{1}{41472} \epsilon^{m_{1} \ldots m_{11}} F_{m_{1} \ldots m_{4}} F_{m_{5} \ldots m_{8}} C_{m_{9} \ldots m_{11}} \\
& +\frac{1}{96} e\left(\bar{\psi}_{n} \Gamma^{m_{1} \ldots m_{4} n l} \psi_{l}+12 \bar{\psi}^{m_{1}} \Gamma^{m_{2} m_{3}} \psi^{m_{4}}\right)(F+\hat{F})_{m_{1} \ldots m_{4}} .
\end{aligned}
$$

where $e=\operatorname{det} e_{m}^{a}$, and $\omega_{m b}^{a}$ denotes the spin connection

$$
\begin{aligned}
\omega_{m b}^{a}= & -e^{n a} \partial_{[m} e_{n] b}+e^{l a} e_{b}^{n} e_{m}^{c} \partial_{[l} e_{n] c}+e_{b}^{n} \partial_{[m} e_{n]}^{a} \\
& +\left(\overline{\psi_{m}} \Gamma_{b} \psi^{a}+\bar{\psi}_{b} \Gamma_{m} \psi^{a}-\overline{\psi_{m}} \Gamma^{a} \psi_{b}\right)-\frac{1}{2} \bar{\psi}_{n} \Gamma_{m b}^{a n p} \psi_{p},
\end{aligned}
$$

and $F_{k l m n}\left(=4 \partial_{[k} C_{l m n]}\right)$ denotes the field strength of the antisymmetric tensor. $R(=$ $\left.e_{b}^{n} e_{a}^{m} R_{m n}{ }^{a b}\right)$ denotes the scalar curvature, where $R_{m n b}^{a}\left(=2 \partial_{[m} \hat{\omega}_{n] b}^{a}-\left[\hat{\omega}_{m}, \hat{\omega}_{n}\right]_{b}^{a}\right)$ denotes curvature tensor.

The derivative $D_{m} \epsilon\left(\equiv\left(\partial_{m}-\frac{1}{4} \omega_{m a b} \Gamma^{a b}\right) \epsilon\right)$ is covariant with respect to local Lorentz transformations. 
The equations of motion are as follows,

$$
\begin{aligned}
R_{m n}(\hat{\omega}) & =\frac{1}{144} g_{m n} \hat{F}_{a b c d} \hat{F}^{a b c d}-\frac{1}{12} \hat{F}_{m a b c} \hat{F}_{n}^{a b c}, \\
0 & =\Gamma^{m n l} \hat{D}_{n}(\hat{\omega}) \psi_{l}, \\
D_{a} \hat{F}^{a b c d} & =-\frac{1}{1152} \epsilon^{b c d a_{1} . a_{8}} \hat{F}_{a_{1} . . a_{4}} \hat{F}_{a_{5} . . a_{8}},
\end{aligned}
$$

where $R_{m n}\left(=e_{n}{ }^{a} e^{l b} R_{m l a b}\right)$ denotes Ricci tensor.

Supersymmetry transformations are equal to

$$
\begin{aligned}
\delta_{s} e_{m}^{a} & =2 \bar{\epsilon} \Gamma^{a} \psi_{m}, \\
\delta_{s} \psi_{m} & =D_{m}(\hat{\omega}) \epsilon+T_{m}^{r s t u} \epsilon \hat{F}_{r s t u} \equiv \hat{D}_{m}(\hat{\omega}) \epsilon, \\
\delta_{s} C_{k l m} & =-6 \bar{\epsilon} \Gamma_{[k l} \psi_{m]}, \\
\text { with, } T_{m}^{r s t u} & \equiv \frac{1}{288}\left(\Gamma_{m}^{r s t u}-8 \delta_{m}^{[r} \Gamma^{s t u]}\right),
\end{aligned}
$$

where $\hat{F}\left(=F_{k l m n}+12 \bar{\psi}_{[k} \Gamma_{l m} \psi_{n]}\right)$ is the supercovariant field strength, and $\hat{\omega}\left(=\omega_{m b}^{a}+\right.$ $\left.\frac{1}{2} \bar{\psi}_{n} \Gamma_{m b}^{a n p} \psi_{p}\right)$ is the supercovariant spin connection.

Note that the spin connection $\omega$ has supersymmetry variation according to elfbein and gravitino's variation in 2nd-order formalism 15. While in 1.5-order formalism, it is defined as a dependent field determined by its equation of motion, whereas its supersymmetry variation is treated as if it were an independent field [16. In this paper we use 2nd-order formalism.

The gauge transformations are equal to

$$
\delta_{c} C_{m n l}=3 \partial_{[m} \xi_{n l]} .
$$

The local Lorentz transformations are equal to

$$
\begin{aligned}
\delta_{l} e_{m}^{a} & =\lambda^{a}{ }_{b} e_{m}^{b}, \\
\delta_{l} \psi_{m}{ }^{\alpha} & =\frac{1}{4} \lambda_{a b} \Gamma^{a b \alpha}{ }_{\beta} \psi_{m}{ }^{\beta}, \\
\delta_{l} \omega_{m b}^{a} & =\partial_{m} \lambda^{a}{ }_{b}+\lambda^{a}{ }_{c} \omega_{m b}{ }^{c}-\omega_{m c}{ }^{a} \lambda^{c}{ }_{b} .
\end{aligned}
$$

The general coordinate transformation are equal to

$$
\begin{aligned}
\delta_{g} e_{m}^{a} & =\xi^{n} \partial_{n} e_{m}^{a}+\partial_{m} \xi^{n} e_{n}^{a}, \\
\delta_{g} \omega_{m b}^{a} & =\xi^{n} \partial_{n} \omega_{m b}^{a}+\partial_{m} \xi^{n} \omega_{n b}^{a}, \\
\delta_{g} \psi_{m}^{a} & =\xi^{n} \partial_{n} \psi_{m}^{a}+\partial_{m} \xi^{n} \psi_{n}^{a} \\
\delta_{g} C_{m n l} & =\xi^{k} \partial_{k} C_{m n l}+3 \partial_{[m} \xi^{k} C_{|k| n l]} .
\end{aligned}
$$


We obtain the full algebra of these transformations as follows

$$
\begin{array}{r}
{\left[\delta_{g}\left(\xi_{1}\right)+\delta_{s}\left(\epsilon_{1}\right)+\delta_{l}\left(\lambda_{1}\right)+\delta_{c}\left(\xi_{1 m n}\right)\right.} \\
\left., \delta_{g}\left(\xi_{2}\right)+\delta_{s}\left(\epsilon_{2}\right)+\delta_{l}\left(\lambda_{2}\right)+\delta_{c}\left(\xi_{2 m n}\right)\right] \\
=\delta_{g}\left(\xi_{3}\right)+\delta_{s}\left(\epsilon_{3}\right)+\delta_{l}\left(\lambda_{3}\right)+\delta_{c}\left(\xi_{3 m n}\right)
\end{array}
$$

where

$$
\begin{aligned}
\xi_{3}^{m}= & \xi_{2}^{n} \partial_{n} \xi_{1}^{m}+\bar{\epsilon}_{2} \Gamma^{m} \epsilon_{1}-(1 \leftrightarrow 2), \\
\epsilon_{3}= & -\bar{\epsilon}_{2} \Gamma^{n} \epsilon_{1} \psi_{n}-\xi_{1}^{n} \partial_{n} \epsilon_{2}+\frac{1}{4} \lambda_{2 c d} \Gamma^{c d} \epsilon_{1}-(1 \leftrightarrow 2), \\
\lambda_{3 b}{ }^{a}= & -\bar{\epsilon}_{2} \Gamma^{n} \epsilon_{1} \hat{\omega}_{n b}^{a}-\xi_{1}^{n} \partial_{n} \lambda_{2}{ }^{a} b+\lambda_{2}{ }^{a}{ }_{c} \lambda_{1 b}{ }^{c} b \\
& +\frac{1}{144} \bar{\epsilon}_{2}\left(\Gamma_{b}^{a}{ }^{r s t u} \hat{F}_{r s t u}+24 \Gamma_{r s} \hat{F}_{b}^{a}{ }_{b}{ }^{r s}\right) \epsilon_{1}-(1 \leftrightarrow 2), \\
\xi_{3 m n}= & -\bar{\epsilon}_{2} \Gamma^{k} \epsilon_{1} C_{k m n}-\bar{\epsilon}_{2} \Gamma_{m n} \epsilon_{1}-\xi_{1}^{k} \partial_{k} \xi_{2 m n}-2 \xi_{1}^{k} \partial_{[m} \xi_{2 n] k} \\
& -(1 \leftrightarrow 2) .
\end{aligned}
$$

\section{Superspace representation}

\subsection{Supermembrane theory}

Supermembrane theory is described as nonlinear sigma model [8]. It is written in terms of superspace embedding coordinates $Z^{M}(\xi)=\left(X^{m}(\xi), \theta(\xi)\right)$, which are functions of the three world-volume coordinate $\xi^{i}(i=0,1,2)$.

The action is

$$
I=\int d^{3} \xi\left(-\frac{1}{2} \sqrt{-g} g^{i j} \Pi_{i}{ }^{a} \Pi_{j}{ }^{b} \eta_{a b}+\frac{1}{2} \sqrt{-g}-\frac{1}{6} \epsilon^{i j k} \Pi_{i}{ }^{A} \Pi_{j}{ }^{B} \Pi_{k}{ }^{C} B_{C B A}\right),
$$

where $g_{i j}$ is the metric of the world-volume, $g=\operatorname{det}\left(g_{i j}\right)$ and $\Pi_{i}{ }^{A} \equiv \partial_{i} Z^{M} E_{M}^{A}$. $E_{M}{ }^{A}$ is supervielbein, and the 3 -form $B=\frac{1}{6} E^{A} E^{B} E^{C} B_{C B A}$ is potential for the closed 4-form $H=d B$.

This action has the following symmetries, world-volume reparametrization $\eta^{i}(\xi)$

$$
\begin{aligned}
\delta Z^{M} & =\eta^{i} \partial_{i} Z^{M} \\
\delta g_{i j} & =\eta^{k} \partial_{k} g_{i j}+2 \partial_{(i} \eta^{k} g_{j) k},
\end{aligned}
$$




$$
\begin{aligned}
\kappa \text {-symmetry } \kappa^{\alpha}(\xi) & \\
\delta Z^{M} E_{M}^{a}= & 0, \\
\delta Z^{M} E_{M}^{\alpha}= & \left(1+\Gamma_{\beta}^{\alpha}\right) \kappa^{\beta}, \\
\delta\left(\sqrt{-g} g^{i j}\right)= & -2\left(1+\Gamma^{\alpha}{ }_{\beta}\right) \kappa^{\beta} \Gamma_{a b \quad \alpha \gamma} \Pi_{n}^{\gamma} g^{n(i} \epsilon^{j) k l} \Pi_{k}{ }^{a} \Pi_{l}{ }^{b} \\
& +\frac{-2}{3 \sqrt{-g}} \kappa^{\alpha} \Gamma_{c \alpha \beta} \Pi^{k \beta} \Pi_{k}^{c} \epsilon^{m n(i} \epsilon^{j) p q} \\
& \left(\Pi_{m}^{a} \Pi_{p a} \Pi_{n}^{b} \Pi_{q b}+\Pi_{m}^{a} \Pi_{p a} g_{n q}+g_{m p} g_{n q}\right),
\end{aligned}
$$

where $\kappa^{\alpha}(\xi)$ is anticommuting space time spinor and the matrix $\Gamma$ is defined by

$$
\Gamma=\frac{1}{6 \sqrt{-g}} \epsilon^{i j k} \Pi_{i}^{a} \Pi_{j}^{b} \Pi_{k}^{c} \Gamma_{a b c} .
$$

Up to surface terms the $\kappa$-invariance of this action imposes the following constraints on the 11-dimensional superspace geometry [9].

$$
\begin{aligned}
T_{\alpha \beta}^{a} & =-2 \Gamma_{\alpha \beta}^{a}, \\
H_{\alpha \beta a b} & =2 \Gamma_{a b \alpha \beta}, \\
H_{\alpha \beta \gamma \delta}=H_{\alpha \beta \gamma d}=H_{\alpha b c d} & =0, \\
T_{\beta \gamma}^{\alpha}=T_{b c}^{a}=T_{b \gamma}^{a} & =0 .
\end{aligned}
$$

If we want interaction terms up to n-th order in anticommuting coordinates in Matrix theory, $E_{m}^{a}$ and $B_{m n l}$ are required up to n-th order in anticommuting coordinates, $E_{\mu}^{a}$ and $B_{\mu m n}$ are required up to (n-1)-th order in anticommuting coordinates, $B_{\mu \nu m}$ is required up to (n-1)-th order in anticommuting coordinates, $B_{\mu \nu \rho}$ is required up to (n-2)-th order in anticommuting coordinates.

\subsection{Superspace formalism}

In this subsection, we explain notations of the superspace geometry and obtain the full algebra of transformations in 11-dimensional superspace. As usual, we suppose that the 11-dimensional superspace has Lorentzian tangent space structure and the vielbein $E_{M}{ }^{A}$ and the connection $\Omega_{M A}{ }^{B}$ and 3-form potential $B$ and its field strength $H$.

The Lorentzian assumption implies

$$
\begin{aligned}
\Omega_{a b} & =-\Omega_{b a}, \\
\Omega_{\alpha b} & =0, \\
\Omega_{\alpha \beta} & =\frac{1}{4} \Omega_{a b} \Gamma^{a b}{ }_{\alpha \beta} .
\end{aligned}
$$


From these basic fields we can define the torsion $\left(T^{A} \equiv D E^{A}=d E^{A}+E^{B} \Omega_{B}^{A}\right)$ and curvature $\left(R_{A}^{B} \equiv d \Omega_{A}^{B}+\Omega_{A}^{C} \Omega_{C}^{B}\right)$. Bianchi identity under constraints (3.5) is as follows,

$$
\begin{aligned}
R_{a d b}{ }^{d} & =\frac{1}{144} \eta_{a b} H_{c d e f} H^{c d e f}-\frac{1}{12} H_{a c d e} H_{b}{ }^{c d e}, \\
0 & =\Gamma_{\beta}^{a \alpha} T_{a b}{ }^{\beta}, \\
D_{a} H^{a b c d} & =-\frac{1}{1152} \epsilon^{b c d a_{1} . . a_{8}} H_{a_{1} . . a_{4}} H_{a_{5} . . a_{8}}, \\
D_{[a} H_{b c d e]} & =0 \\
R_{[a b c] d}=R_{a[b c d]} & =0 \\
R_{\alpha \beta a b} & =-\frac{1}{3} H_{a b c d} \Gamma_{\alpha \beta}^{c d}-\frac{1}{72} H_{c d e f} \Gamma_{a b}{ }^{c d e f}{ }_{\alpha \beta}, \\
R_{\beta d c a} & =T_{c d} \Gamma_{a \epsilon \beta}+T_{d a} \Gamma_{c \epsilon \beta}-T_{a c} \Gamma_{d \epsilon \beta}, \\
T_{a \beta}{ }^{\alpha} & =\frac{1}{36} H_{a b c d} \Gamma^{b c d \alpha}{ }_{\beta}-\frac{1}{288} H_{b c d e} \Gamma_{a}{ }_{a c d e \alpha}{ }_{\beta} \\
D^{\alpha} H_{a b c d} & =-12 \Gamma_{[a b \beta}{ }_{\alpha} T_{c d]}{ }_{\beta}-\frac{1}{7}\left(\Gamma_{[a b} \Gamma^{e f} D H_{c d] e f}\right)^{\alpha} .
\end{aligned}
$$

The supertransformation is equal to

$$
\delta_{T} X_{M_{p} \ldots M_{1}}=\Xi^{K} \partial_{K} X_{M_{p} \ldots M_{1}}+p \partial_{\left[M_{p}\right.} \Xi^{K} X_{\left.|K| M_{p-1} \ldots M_{1}\right]}
$$

for p-form's components. The local Lorentz transformations are equal to

$$
\begin{aligned}
\delta_{L} E^{A} & =E^{B} \Lambda_{B}{ }^{A}, \\
\delta_{L} \Omega_{B}{ }^{A} & =-\Lambda_{B}{ }^{C} \Omega_{C}{ }^{A}+\Omega_{B}{ }^{C} \Lambda_{C}{ }^{A}-d \Lambda_{B}{ }^{A} .
\end{aligned}
$$

The supergauge transformations are equal to

$$
\delta_{G} B_{L M N}=3 \partial_{[L} \Xi_{M N]} .
$$

We obtain the full algebra of these transformations as follows

$$
\begin{array}{r}
{\left[\delta_{T}\left(\Xi_{1}\right)+\delta_{L}\left(\Lambda_{1}\right)+\delta_{G}\left(\Xi_{1 M N}\right), \delta_{T}\left(\Xi_{2}\right)+\delta_{L}\left(\Lambda_{2}\right)+\delta_{G}\left(\Xi_{2 M N}\right)\right]} \\
=\delta_{T}\left(\Xi_{3}\right)+\delta_{L}\left(\Lambda_{3}\right)+\delta_{G}\left(\Xi_{3 M N}\right),
\end{array}
$$

where,

$$
\begin{aligned}
\Xi_{3}^{K} & =\Xi_{2}^{L} \partial_{L} \Xi_{1}^{K}+\delta_{1} \Xi_{2}^{K}-(1 \leftrightarrow 2), \\
\Lambda_{3 A}{ }^{B} & =-\Xi_{1}^{K} \partial_{K} \Lambda_{2 A}{ }^{B}+\delta_{1} \Lambda_{2 A}{ }^{B}+\Lambda_{1 A}{ }^{C} \Lambda_{2 C}{ }^{B}-(1 \leftrightarrow 2), \\
\Xi_{3 M N} & =\delta_{1} \Xi_{2 M N}-\Xi_{1}^{K} \partial_{K} \Xi_{2 M N}-2 \partial_{[M} \Xi_{2 N] K} \Xi_{1}^{K}-(1 \leftrightarrow 2) .
\end{aligned}
$$


There are a great number of component fields in superspace. Thus if we try to identify superspace representation as ordinary supergravity, there are a great number of unknown degrees of freedom. The method of this identification is known as 'gauge completion' [17. We shall explain it in the next section.

\section{Gauge completion}

'Gauge completion' was introduced to identify superspace representation as on-shell supergravity [17]. In this section we review this method and calculate part of components of the superfield in terms of the on-shell supergravity fields.

Using this method, up to first order in anticommuting coordinates, the superfield components was investigated by E. Cremmer and S. Ferrara [9]. Part of components at second order in anticommuting coordinates was investigated by B. de Wit, K. Peeters and J. Plefka [0].

\subsection{Gauge completion procedure}

'Gauge completion' is searching for structures of the superfields and superparameters which are compatible with ordinary supergravity. That is to say, supertransformations

(3.16) - (3.18) are identified as transformations in 11-dimensional spacetime (2.6), (2.8), (2.9), (2.10) and the $\theta=0$ components of superfields and superparameters are identified as the fields and parameters of ordinary supergravity.

Firstly, we choose the input data as follows

$$
\begin{aligned}
E_{m}^{a(0)} & =e_{m}^{a}, \\
E_{m}^{\alpha(0)} & =\psi_{m}^{\alpha}, \\
\Omega_{m b}^{a(0)} & =-\hat{\omega}_{m b}^{a}, \\
\Xi^{m(0)} & =\xi^{m}, \\
\Xi^{\mu(0)} & =\epsilon^{\mu}, \\
\Xi_{m n}^{(0)} & =\xi_{m n}, \\
B_{m n l}^{(0)} & =C_{m n l} .
\end{aligned}
$$

From (3.17) and (2.9), we obtain

$$
\Lambda_{b}{ }^{a(0)}=\lambda^{a}{ }_{b} .
$$


Moreover we introduce the assumption that superparameters do not include the derivative of $\epsilon$. Then, the higher order components in anticommuting coordinates can be obtained by requiring consistency between the algebra of superspace supergravity and that of ordinary supergravity.

If we can represent $\Xi_{M N}=2 \partial_{[M} \Phi_{N]}$, we can choose the gauge as $\Xi_{M N}=0$ because this superparameters do not change the 3-form superfields (3.18) and the algebra (3.20). Thus we can choose the gauge as follows,

$$
\Xi_{\mu N}^{(0)}=0
$$

To obtain the higher order components of superparameters which depend on $\epsilon$, we must calculate the commutation of two supersymmetry transformation.

According to (2.12), (3.20) and (4.14),

$$
\begin{aligned}
{\left[\delta_{s 1}, \delta_{s 2}\right] E_{m}{ }^{a(0)}=} & \left.\left(\Xi_{3}^{K} \partial_{K} E_{m}^{a}+\partial_{m} \Xi_{3}^{K} E_{K}^{a}+E_{m}^{b} \Lambda_{3 b}{ }^{a}\right)\right|_{\theta=0} \\
= & \left(\delta_{g}\left(2 \bar{\epsilon}_{2} \Gamma^{m} \epsilon_{1}\right)+\delta_{s}\left(-2 \bar{\epsilon}_{2} \Gamma^{n} \epsilon_{1} \psi_{n}\right)+\delta_{c}\left(-2 \bar{\epsilon}_{2} \Gamma^{k} \epsilon_{1} C_{k m n}-2 \bar{\epsilon}_{2} \Gamma_{m n} \epsilon_{1}\right)\right. \\
& \left.+\delta_{l}\left(-2 \bar{\epsilon}_{2} \Gamma^{n} \epsilon_{1} \hat{\omega}_{n b}^{a}+\frac{1}{72} \bar{\epsilon}_{2}\left(\Gamma_{b}^{a}{ }_{b}^{r s t u} \hat{F}_{r s t u}+24 \Gamma_{r s} \hat{F}_{b}^{a}{ }^{r s}\right) \epsilon_{1}\right)\right) e_{m}^{a} .
\end{aligned}
$$

Thus one obtains

$$
\Xi^{k(1)}(\text { susy })=\bar{\theta} \Gamma^{k} \epsilon
$$

In the same way, to obtain the higher order components of superparameters which depend on $\lambda$ we must calculate the commutation of supersymmetry transformation and Lorentz transformation. To obtain the higher order components of superparameters which depend on $\xi_{m n}$ we must calculate the commutation of supersymmetry transformation and gauge transformation. To obtain the higher order components of superparameters which depend on $\xi^{m}$ we must calculate the commutation of supersymmetry transformation and general coordinate transformation.

By this procedure, the following results had been known [9], [7].

$$
\begin{aligned}
\Xi^{m} & =\xi^{m}+\bar{\theta} \Gamma^{m} \epsilon-\bar{\theta} \Gamma^{n} \epsilon \bar{\theta} \Gamma^{m} \psi_{n}+\mathcal{O}\left(\theta^{3}\right) \\
\Xi^{\mu} & =\epsilon^{\mu}-\frac{1}{4} \lambda_{c d}\left(\Gamma^{c d} \theta\right)^{\mu}-\bar{\theta} \Gamma^{n} \epsilon \psi_{n}^{\mu}+\mathcal{O}\left(\theta^{2}\right) \\
\Lambda_{b}{ }^{a} & =\lambda_{b}^{a}-\bar{\theta} \Gamma^{n} \epsilon \hat{\omega}_{n b}^{a}+\frac{1}{144} \bar{\theta}\left(\Gamma_{b}^{a}{ }^{r s t u} \hat{F}_{r s t u}+24 \Gamma_{r s} \hat{F}_{b}^{a}{ }^{r s}\right) \epsilon+\mathcal{O}\left(\theta^{2}\right) \\
\Xi_{m n} & =\xi_{m n}-\left(\bar{\theta} \Gamma^{p} \epsilon C_{p m n}+\bar{\theta} \Gamma_{m n} \epsilon\right)+\bar{\theta} \Gamma^{k} \epsilon \bar{\theta} \Gamma^{l} \psi_{k} C_{l m n}+\bar{\theta} \Gamma^{k} \epsilon \bar{\theta} \Gamma_{m n} \psi_{k}+\frac{4}{3} \bar{\theta} \Gamma^{l} \epsilon \bar{\theta} \Gamma_{l[m} \psi_{n]}
\end{aligned}
$$




$$
\begin{aligned}
& +\frac{4}{3} \bar{\theta} \Gamma^{l} \psi_{[n} \bar{\theta} \Gamma_{|l| m]} \epsilon+\mathcal{O}\left(\theta^{3}\right) \\
\Xi_{m \mu}= & \frac{1}{6} \bar{\theta} \Gamma^{n} \epsilon\left(\bar{\theta} \Gamma_{m n}\right)_{\mu}+\frac{1}{6}\left(\bar{\theta} \Gamma^{n}\right)_{\mu} \bar{\theta} \Gamma_{m n} \epsilon+\mathcal{O}\left(\theta^{3}\right), \\
\Xi_{\mu \nu}= & \mathcal{O}\left(\theta^{3}\right) .
\end{aligned}
$$

According to superspace algebra,

$$
\begin{aligned}
\left.\delta_{\text {susy }} E_{m}{ }^{a}\right|_{\theta=0} & =\left.\left(\Xi^{K}(\text { susy }) \partial_{K} E_{m}^{a}+\partial_{m} \Xi^{K}(\text { susy }) E_{K}^{a}+E_{m}^{b} \Lambda_{b}{ }^{a}(\text { susy })\right)\right|_{\theta=0} \\
& =\epsilon^{\nu} \partial_{\nu}\left(E_{m}^{a(1)}\right)+\partial_{m} \epsilon^{\nu} E_{\nu}{ }^{a(1)},
\end{aligned}
$$

while in ordinary supergravity

$$
\delta_{\text {susy }} e_{m}^{a}=2 \bar{\epsilon} \Gamma^{a} \psi_{m}
$$

Thus, one obtains

$$
\begin{aligned}
E_{\nu}{ }^{a(0)} & =0, \\
E_{m}{ }^{a(1)} & =2 \bar{\theta} \Gamma^{a} \psi_{m} .
\end{aligned}
$$

By this procedure, the following results had been known [9], [7].

$$
\begin{aligned}
E_{m}^{a}= & e_{m}^{a}+2 \bar{\theta} \Gamma^{a} \psi_{m}-\frac{1}{4} \bar{\theta} \Gamma^{a c d} \theta \hat{\omega}_{m c d}+\frac{1}{72} \bar{\theta} \Gamma_{m}^{r s t} \theta \hat{F}_{r s t}{ }^{a} \\
& +\frac{1}{288} \bar{\theta} \Gamma^{r s t u} \theta \hat{F}_{r s t u} e_{m}^{a}-\frac{1}{36} \bar{\theta} \Gamma^{a s t u} \theta \hat{F}_{m s t u}+\mathcal{O}\left(\theta^{3}\right), \\
E_{m}^{\alpha}= & \psi_{m}^{\alpha}-\frac{1}{4} \hat{\omega}_{m a b}\left(\Gamma^{a b} \theta\right)^{\alpha}+\left(T_{m}^{r s t u} \theta\right)^{\alpha} \hat{F}_{r s t u}+\mathcal{O}\left(\theta^{2}\right), \\
E_{\mu}{ }^{a}= & -\left(\Gamma^{a} \theta\right)_{\mu}+\mathcal{O}\left(\theta^{3}\right), \\
E_{\mu}{ }^{\alpha}= & \delta_{\mu}^{\alpha}+\mathcal{O}\left(\theta^{2}\right), \\
\Omega_{\mu b}{ }^{a}= & \frac{1}{144}\left\{\left(\Gamma_{b}^{a}{ }_{b}^{r s t u} \theta\right)_{\mu} \hat{F}_{r s t u}+24\left(\Gamma_{r s} \theta\right)_{\mu} \hat{F}_{b}^{a}{ }^{r s}\right\}+\mathcal{O}\left(\theta^{2}\right), \\
\Omega_{m a b}= & \hat{\omega}_{m a b}+2 \bar{\theta}\left\{e^{n} e^{k}{ }_{b}\left(-\Gamma_{k} D_{[m} \psi_{n]}+\Gamma_{n} D_{[m} \psi_{k]}+\Gamma_{m} D_{[n} \psi_{k]}\right)\right\} \\
& +\frac{1}{72} \bar{\theta}\left(\Gamma_{a b}{ }^{r s t u} \hat{F}_{r s t u}+24 \Gamma_{r s} \hat{F}_{a b}{ }^{r s}\right) \psi_{m}+\mathcal{O}\left(\theta^{2}\right), \\
B_{m n l}= & C_{m n l}-6 \bar{\theta} \Gamma_{[m n} \psi_{l]}+\frac{3}{4} \hat{\omega}_{[l}{ }^{c d} \bar{\theta} \Gamma_{m n] c d} \theta-\frac{3}{2} \hat{\omega}_{[l m n]} \theta^{2} \\
& -\frac{1}{96} \bar{\theta} \Gamma_{m n l}^{r s t u} \theta \hat{F}_{r s t u}-\frac{3}{8} \bar{\theta} \Gamma_{[l}{ }^{r s} \theta \hat{F}_{|r s| m n]}-12 \bar{\theta} \Gamma_{a} \psi_{[m} \bar{\theta} \Gamma_{n}^{a} \psi_{l]}
\end{aligned}
$$




$$
\begin{aligned}
& +\mathcal{O}\left(\theta^{3}\right), \\
B_{m n \mu}= & \left(\bar{\theta} \Gamma_{m n}\right)_{\mu}+\frac{8}{3} \bar{\theta} \Gamma^{k} \psi_{[m}\left(\bar{\theta} \Gamma_{|k| n]}\right)_{\mu}+\frac{4}{3}\left(\bar{\theta} \Gamma^{k}\right)_{\mu} \bar{\theta} \Gamma_{k[m} \psi_{n]}+\mathcal{O}\left(\theta^{3}\right), \\
B_{m \mu \nu}= & \left(\bar{\theta} \Gamma_{m n}\right)_{(\mu}\left(\bar{\theta} \Gamma^{n}\right)_{\nu)}+\mathcal{O}\left(\theta^{3}\right) \\
B_{\mu \nu \rho}= & \left(\bar{\theta} \Gamma_{m n}\right)_{(\mu}\left(\bar{\theta} \Gamma^{m}\right)_{\nu}\left(\bar{\theta} \Gamma^{n}\right)_{\rho)}+\mathcal{O}\left(\theta^{3}\right) .
\end{aligned}
$$

Because the flat geometry had been known, we include the $\theta^{3}$ term in $B_{\mu \nu \rho}$ for completeness.

\subsection{Calculation}

$\Xi^{\mu(2)}$ is subject to the following equations,

$$
\begin{aligned}
\epsilon_{2}^{\nu} \partial_{\mu} \partial_{\nu} \Xi_{1}^{\alpha}-(1 \leftrightarrow 2)= & \frac{1}{576} \epsilon_{2}^{\nu}\left(\Gamma_{a b}{ }^{r s t u}+24 \delta_{b}{ }^{u} \delta_{a}{ }^{t} \Gamma^{r s} \epsilon_{1}\right)_{\nu} \Gamma^{a b \alpha}{ }_{\mu} \hat{F}_{r s t u} \\
& +\left(\Gamma^{k} \epsilon_{1}\right)_{\mu}\left(T_{k}^{r s t u}\right)_{\nu}^{\alpha} \epsilon_{2}^{\nu} \hat{F}_{r s t u}-\epsilon_{2}^{\nu}\left(\Gamma^{n} \epsilon_{1}\right)_{\nu}\left(\Gamma^{k} \psi_{n}\right)_{\mu} \psi_{k}{ }^{\alpha} \\
& -\left(\Gamma^{n} \epsilon_{1}\right)_{\mu} \epsilon_{2}^{\nu}\left(\Gamma^{k} \psi_{n}\right)_{\nu} \psi_{k}{ }^{\alpha}-\frac{1}{4}\left(\Gamma^{n} \epsilon_{1}\right)_{\mu} \hat{\omega}_{n a b}\left(\Gamma^{a b}\right)_{\nu}^{\alpha} \epsilon_{2}^{\nu} \\
& -\frac{1}{4} \epsilon_{2}^{\nu}\left(\Gamma^{n} \epsilon_{1}\right)_{\nu} \hat{\omega}_{n a b}\left(\Gamma^{a b}\right)^{\alpha}{ }_{\mu}-(1 \leftrightarrow 2) .
\end{aligned}
$$

However, if simply we drive the equation,

$$
\begin{aligned}
\epsilon_{2}^{\nu} \partial_{\mu} \partial_{\nu} \Xi_{1}^{\alpha}= & \left(\Gamma^{k} \epsilon_{1}\right)_{\mu}\left(T_{k}^{r s t u}\right)_{\nu}^{\alpha} \epsilon_{2}^{\nu} \hat{F}_{r s t u}-\epsilon_{2}^{\nu}\left(\Gamma^{n} \epsilon_{1}\right)_{\nu}\left(\Gamma^{k} \psi_{n}\right)_{\mu} \psi_{k}{ }^{\alpha} \\
& +\frac{1}{576} \epsilon_{2}^{\nu}\left(\Gamma_{a b}{ }^{r s t u}+24 \delta_{b}{ }^{u} \delta_{a}{ }^{t} \Gamma^{r s} \epsilon_{1}\right)_{\nu} \Gamma^{a b \alpha}{ }_{\mu} \hat{F}_{r s t u} \\
& -\left(\Gamma^{n} \epsilon_{1}\right)_{\mu} \epsilon_{2}^{\nu}\left(\Gamma^{k} \psi_{n}\right)_{\nu} \psi_{k}{ }^{\alpha}-\frac{1}{4}\left(\Gamma^{n} \epsilon_{1}\right)_{\mu} \hat{\omega}_{n a b}\left(\Gamma^{a b}\right)^{\alpha}{ }_{\nu} \epsilon_{2}^{\nu} \\
& -\frac{1}{4} \epsilon_{2}^{\nu}\left(\Gamma^{n} \epsilon_{1}\right)_{\nu} \hat{\omega}_{n a b}\left(\Gamma^{a b}\right)^{\alpha}{ }_{\mu},
\end{aligned}
$$

this equation is inconsistent because $\mu$ and $\nu$ in the left-hand side of it are antisymmetric but these in the right-hand side of it are not antisymmetric. Thus we must add terms in the right-hand side of this equation.

$$
\epsilon_{2}^{\nu} \partial_{\mu} \partial_{\nu} \Xi_{1}^{\alpha}=\left(\Gamma^{k} \epsilon_{1}\right)_{\mu}\left(T_{k}^{r s t u} \epsilon_{2}\right)^{\alpha} \hat{F}_{r s t u}+\frac{1}{576} \bar{\epsilon}_{2}\left(\Gamma_{a b}{ }^{r s t u}+24 \delta_{b}^{u} \delta_{a}{ }^{t} \Gamma^{r s}\right) \epsilon_{1} \Gamma_{\mu}^{a b \alpha}{ }_{\mu} \hat{F}_{r s t u}
$$




$$
\begin{aligned}
& +\frac{\hat{F}_{r s t u}}{576}\left[2 \bar{\epsilon}_{2} \Gamma^{c b u} \epsilon_{1} \Gamma_{b c}{ }^{r s t}-6 \bar{\epsilon}_{2} \Gamma^{c t u} \epsilon_{1} \Gamma_{c}^{r s}-3 \bar{\epsilon}_{2} \Gamma^{d c t u} \epsilon_{1} \Gamma_{c d}{ }^{r s}-4 \bar{\epsilon}_{2} \Gamma^{d s t u} \epsilon_{1} \Gamma_{d}^{r}\right. \\
& \left.+8 \bar{\epsilon}_{2} \Gamma^{r s t u} \epsilon_{1} \delta\right]_{\mu}^{\alpha}-\epsilon_{2}^{\nu}\left(\Gamma^{n} \epsilon_{1}\right)_{\nu}\left(\Gamma^{k} \psi_{n}\right)_{\mu} \psi_{k}^{\alpha}-\frac{1}{4} \epsilon_{2}^{\nu}\left(\Gamma^{n} \epsilon_{1}\right)_{\nu} \hat{\omega}_{n a b}\left(\Gamma^{a b}\right)_{\mu}^{\alpha} \\
& -\left(\Gamma^{n} \epsilon_{1}\right)_{\mu} \epsilon_{2}^{\nu}\left(\Gamma^{k} \psi_{n}\right)_{\nu} \psi_{k}{ }^{\alpha}-\frac{1}{4}\left(\Gamma^{n} \epsilon_{1}\right)_{\mu} \hat{\omega}_{n a b}\left(\Gamma^{a b}\right)_{\nu}^{\alpha} \epsilon_{2}^{\nu} .
\end{aligned}
$$

Thus we obtain

$$
\begin{aligned}
\Xi^{\mu}= & \hat{F}_{a b c d}\left[-\frac{1}{1024} \bar{\theta} \theta\left(\Gamma^{a b c d} \epsilon\right)^{\mu}+\bar{\theta} \Gamma^{e f g} \theta\left(\frac{5}{18432}\left(\Gamma_{g f e}{ }^{a b c d} \epsilon\right)^{\mu}-\frac{7}{4608} \delta_{g}{ }^{d}\left(\Gamma_{f e}{ }^{a b c} \epsilon\right)^{\mu}\right.\right. \\
& \left.-\frac{5}{1536} \delta_{g}{ }^{d} \delta_{f}{ }^{c}\left(\Gamma_{e}{ }^{a b} \epsilon\right)^{\mu}+\frac{7}{768} \delta_{g}{ }^{d} \delta_{f}{ }^{c} \delta_{e}{ }^{b}\left(\Gamma^{a} \epsilon\right)^{\mu}\right)+\bar{\theta} \Gamma^{e f g h} \theta\left(\frac{5}{73728}\left(\Gamma_{h g f e}{ }^{a b c d} \epsilon\right)^{\mu}\right. \\
& +\frac{1}{4608} \delta_{h}{ }^{d}\left(\Gamma_{g f e}{ }^{a b c} \epsilon\right)^{\mu}+\frac{1}{1024} \delta_{h}{ }^{d} \delta_{g}{ }^{c}\left(\Gamma_{f e}{ }^{a b} \epsilon\right)^{\mu}-\frac{1}{2304} \delta_{h}{ }^{d} \delta_{g}{ }^{c} \delta_{f}{ }^{b}\left(\Gamma_{e}{ }^{a} \epsilon\right)^{\mu} \\
& \left.\left.-\frac{73}{9216} \delta_{h}{ }^{d} \delta_{g}{ }^{c} \delta_{f}{ }^{b} \delta_{e}{ }^{a} \epsilon^{\mu}\right)\right]+\bar{\theta} \Gamma^{n} \epsilon \bar{\theta} \Gamma^{k} \psi_{n} \psi_{k}{ }^{\mu}+\frac{1}{4} \bar{\theta} \Gamma^{n} \epsilon \hat{\omega}_{n a b}\left(\Gamma^{a b} \theta\right)^{\mu}
\end{aligned}
$$

$E_{\mu}{ }^{\alpha(2)}$ is subject to the following equation,

$$
\begin{aligned}
\epsilon^{\nu} \partial_{\nu} E_{\mu}{ }^{\alpha(2)}= & -\partial_{\mu} \Xi^{\alpha(2)}(\hat{F} \text { dependent terms })-\left(\Gamma^{k} \epsilon\right)_{\mu}\left(T_{k}{ }^{r s t u} \theta\right)^{\alpha} \hat{F}_{r s t u} \\
& -\frac{\hat{F}_{r s t u}}{576} \bar{\theta}\left(\Gamma_{a b}{ }^{r s t u}+24 \delta_{b}{ }^{u} \delta_{a}{ }^{t} \Gamma^{r s}\right) \epsilon \Gamma_{\mu}^{a b \alpha} .
\end{aligned}
$$

From (4.29) we obtain

$$
\begin{aligned}
E_{\mu}^{\alpha}= & \hat{F}_{a b c d}\left[\bar{\theta} \Gamma^{e f g} \theta\left(\frac{1}{576} \delta_{g}{ }^{d} \Gamma_{f e}{ }^{a b c \alpha}{ }_{\mu}-\frac{293}{55296} \delta_{g}{ }^{d} \delta_{f}{ }^{c} \Gamma_{e}{ }^{a b \alpha}{ }_{\mu}\right)\right. \\
& +\bar{\theta} \Gamma^{e f g h} \theta\left(-\frac{1}{384} \delta_{h}{ }^{d} \delta_{g}{ }^{c} \Gamma_{f e}{ }^{a b \alpha}{ }_{\mu}-\frac{1}{288} \delta_{h}{ }^{d} \delta_{g}{ }^{c} \delta_{f}{ }^{b} \Gamma_{e}{ }^{a \alpha}{ }_{\mu}\right. \\
& \left.\left.+\frac{1}{144} \delta_{h}{ }^{d} \delta_{g}{ }^{c} \delta_{f}{ }^{b} \delta_{e}{ }^{a} \delta^{\alpha}{ }_{\mu}\right)\right] .
\end{aligned}
$$

$E_{m}^{\alpha(2)}$ is subject to the following equation,

$$
\begin{aligned}
\epsilon^{\mu} \partial_{\mu} E_{m}^{\alpha}= & \bar{\theta} \Gamma^{k} \epsilon\left(T_{k}{ }^{r s t u} \psi_{m}\right)^{\alpha} \hat{F}_{r s t u}-\frac{\hat{F}_{r s t u}}{576} \bar{\theta}\left(\Gamma_{a b}^{r s t u}+24 \delta_{a}^{r} \delta_{b}^{s} \Gamma^{t u}\right) \epsilon\left(\Gamma^{a b} \psi_{m}\right)^{\alpha} \\
& -\frac{\hat{F}_{r s t u}}{288} \bar{\epsilon}\left(\Gamma_{a b}^{r s t u}+24 \delta_{a}^{r} \delta_{b}^{s} \Gamma^{t u}\right) \psi_{m}\left(\Gamma^{a b} \theta\right)^{\alpha}+2 \bar{\epsilon} \Gamma^{k} \psi_{m}\left(T_{k}{ }^{r s t u} \theta\right)^{\alpha} \hat{F}_{r s t u} \\
& -2 \bar{\epsilon} \Gamma^{k} \theta\left(\hat{D}_{[m} \psi_{n]}\right)^{\alpha}-24\left(T_{m}^{r s t u} \theta\right)^{\alpha} \bar{\epsilon} \Gamma_{[r s} \hat{D}_{t} \psi_{u]}+\bar{\epsilon} \Gamma_{b} \hat{D}_{[m} \psi_{n]}\left(\Gamma^{n b} \theta\right)^{\alpha} \\
& -\frac{1}{2} \bar{\epsilon} \Gamma_{m} \hat{D}_{[l} \psi_{n]}\left(\Gamma^{l n} \theta\right)^{\alpha} .
\end{aligned}
$$


We obtain

$$
\begin{aligned}
E_{m}^{\alpha}= & \bar{\theta} \theta\left[-\frac{1}{384}\left(\Gamma^{a b c d} \psi_{m}\right)^{\alpha} \hat{F}_{a b c d}\right]+\bar{\theta} \Gamma^{e f g} \theta\left[\left(\frac{1}{2304} \Gamma_{g f e}{ }^{a b c d}+\frac{1}{576} \delta_{g}{ }^{d} \Gamma_{f e}{ }^{a b c}\right.\right. \\
& \left.-\frac{1}{192} \delta_{g}{ }^{d} \delta_{f}{ }^{c} \Gamma_{e}{ }^{a b}+\frac{1}{96} \delta_{g}{ }^{d} \delta_{f}{ }^{c} \delta_{e}{ }^{b} \Gamma^{a}\right)^{\alpha}{ }_{\beta} \psi_{m}{ }^{\beta} \hat{F}_{a b c d}+\frac{1}{8}\left(\Gamma_{f e} D_{[m} \psi_{g]}\right)^{\alpha} \\
& \left.+\frac{1}{4} e_{m g}\left(D_{f} \psi_{e}\right)^{\alpha}\right]+\bar{\theta} \Gamma^{e f g h} \theta\left[\left(\frac{1}{27648} \Gamma_{h g f e}{ }^{a b c d}+\frac{1}{1728} \delta_{h}{ }^{d} \Gamma_{g f e}{ }^{a b c}\right.\right. \\
& -\frac{1}{384} \delta_{h}{ }^{d} \delta_{g}{ }^{c} \Gamma_{f e}{ }^{a b}-\frac{1}{288} \delta_{h}{ }^{d} \delta_{g}{ }^{c} \delta_{f}{ }^{b} \Gamma_{e}{ }^{a} \\
& \left.-\frac{1}{1152} \delta_{h}{ }^{d} \delta_{g}{ }^{c} \delta_{f}{ }^{b} \delta_{e}{ }^{a} \delta\right)^{\alpha}{ }_{\beta} \psi_{m}{ }^{\beta} \hat{F}_{a b c d}-\frac{1}{24}\left(\Gamma_{g f e} D_{[m} \psi_{h]}\right)^{\alpha}-\frac{1}{24}\left(\Gamma_{h g m} D_{f} \psi_{e}\right)^{\alpha} \\
& \left.+\frac{1}{6} e_{m h}\left(\Gamma_{g} D_{f} \psi_{e}\right)^{\alpha}\right] .
\end{aligned}
$$

Thus, we have obtained all components of vielbein superfields and 3-form superfields up to second order in anticommuting coordinates.

This results are consistent with the all transformations calculation.

\subsection{Bianchi and constraints}

From results of the previous subsection, we obtain the torsion fields and field strength fields as follows,

$$
\begin{aligned}
T_{\alpha \beta}^{a(0)} & =-2 \Gamma_{\alpha \beta}^{a}, \\
H_{\alpha \beta a b}^{(0)} & =2 \Gamma_{a b \alpha \beta}, \\
H_{\alpha \beta \gamma \delta}^{(0)}=H_{\alpha \beta \gamma d}^{(0)}=H_{\alpha b c d}^{(0)} & =0, \\
T_{\beta \gamma}^{\alpha(0)}=T_{b c}^{a(0)}=T_{b \gamma}^{a(0)} & =0, \\
T_{c b}{ }^{\alpha(0)} & =2 e_{b}^{m} e_{c}^{n} \hat{D}_{[n} \psi_{m]}^{\alpha}, \\
T_{c \beta}^{\alpha(0)} & =\frac{1}{36} \hat{F}_{c f g h} \Gamma_{\beta}^{f g h \alpha}-\frac{1}{288} \hat{F}_{e f g h} \Gamma_{c}{ }^{e f g h \alpha}{ }_{\beta}, \\
H_{a b c d}^{(0)} & =\hat{F}_{a b c d} .
\end{aligned}
$$

This results satisfy the $\kappa$-symmetry constraints (3.5). By using the Bianchi identity (3.7),

we obtain equations of motion in component formalism (2.3). In particular, the equation of motion for gravitino fields can be obtained by using gauge fixing condition,

$$
\begin{aligned}
\left(\hat{D}^{m} \psi_{m}\right)^{\alpha} & =0 \\
\left(\Gamma^{m} \psi_{m}\right)^{\alpha} & =0 .
\end{aligned}
$$


Thus this configuration can be identified as backgrounds for supermembrane.

\section{Discussion}

We have obtained $\Xi^{\mu(2)}, E_{M}^{\alpha(2)}$. Up to second order in anticommuting coordinates, $\Lambda_{a}^{b(2)}$ and $\Omega_{M A}{ }^{B(2)}$ remain. These terms and terms which are required to obtain terms of Matrix theory which are third order in anticommuting coordinates is under considerations. The background field linear coupling to flat Matrix in all order of anticommuting coordinates was conjectured in ref. [18].

There is a problem of interest to us. It is a gauge fixing problem. As a previous section, gravitino fields are subject to gauge fixing conditions (4.41). Whether for $e_{m}^{a}, c_{l m n}$ gauge fixing conditions are required or not, it is not yet obvious. From the beginning the supermembrane theory has general coordinates transformation symmetry, local supersymmetry, local Lorentz symmetry and $U(1)$ gauge symmetry, however to holds $\kappa$-symmetry, we have the constraints for backgrounds which contain gravitino's gauge fixing conditions. I think interpretation of $\kappa$-symmetry must be investigated further.

\section{Acknowledgments}

I would like to thank Y.Matsuo for valuable suggestions, and thank K. Hosomichi for valuable comments and discussions.

\section{Appendix}

\section{A Indices}

We use Greek indices for spinorial components and Latin indices for vector components. And we use former alphabet for the tangent space indices and later for general coordinates indices: $a, b, c, \ldots$ for tangent vector indices and $k, l, m, \ldots$ for general vector indices, and $\alpha, \beta, \ldots$ for tangent spinorial indices and $\mu, \nu, \ldots$ for general spinorial indices.

Superspace coordinates $\left(x^{m}, \theta^{\mu}\right)$ are designated $Z^{M}$, where later capital Latin alphabet $M, N, .$. are collective designations for general coordinate indices. While former capital Latin alphabet $A, B,$. are collective designations for tangent space indices. 


\section{B p-form superfield}

We introduce p-form superfields as follows,

$$
\begin{aligned}
X & \equiv \frac{1}{p !} d z^{M_{p}} \ldots d z^{M_{1}} X_{M_{p} \ldots M_{1}} \\
& \equiv \frac{1}{p !} E^{A_{p}} \ldots E^{A_{1}} X_{A_{p} \ldots A_{1}} \\
X_{A_{p} \ldots A_{1}} & \equiv \sum_{i=1}^{32} X_{A_{p} \ldots A_{1}}{ }^{(i)}
\end{aligned}
$$

$X_{A_{p} \ldots A_{1}}{ }^{(i)}$ is component at $\mathrm{i}$-th order in anticommuting coordinates.

\section{Convention}

Symmetrization bracket ( ) and antisymmetrization bracket [ ] is defined as follows,

$$
\begin{aligned}
& {\left[M_{1} \ldots M_{N}\right]=\frac{1}{N !}\left(M_{1} \ldots M_{N}+\text { antisymmetric terms }\right)} \\
& \left(M_{1} \ldots M_{N}\right)=\frac{1}{N !}\left(M_{1} \ldots M_{N}+\text { symmetric terms }\right) .
\end{aligned}
$$

\section{Gamma matrices(11-dimensional)}

Since we use the Majorana representation, all components are real.

Gamma matrix $\Gamma^{a}{ }_{\beta}$ is defined as follows,

$$
\left\{\Gamma^{a}, \Gamma^{b}\right\}=2 \eta^{a b}
$$

We use the mostly plus metric; $\eta_{a b} \sim(-+\ldots+)$. We lower the spinorial indices by charge conjugation matrix $C_{\alpha \beta}$.

$$
\begin{array}{r}
\bar{\psi}_{\beta}=\psi^{\alpha} C_{\alpha \beta}, \\
\Gamma_{\alpha \beta}^{a}=C_{\alpha \gamma} \Gamma_{\beta}^{a \gamma} .
\end{array}
$$

$\Gamma_{\alpha \beta}^{a_{1} . . a_{n}}(n=1,2,5,6,9,10)$ are symmetric matrices and $\Gamma_{\alpha \beta}^{a_{1} . . a_{n}}(n=0,3,4,7,8,11)$ are antisymmetric matrices. 


\section{References}

[1] T. Banks, W. Fischler, S. H. Shenker and L. Susskind, Phys. Rev. D55, 5112 (1997).

[2] E. Witten, Nucl.Phys. B460, 335 (1996).

[3] B. Bigatti and L. Susskind, hep-th/9712072;

T. Banks, Nucl. Phys. Proc. suppl. 67, 180 (1998).

[4] Y. Okawa and T. Yoneya, Nucl. Phys. B538, 67 (1999).

[5] R. G. Leigh, Mod. Phys. Lett. A4, 2767 (1989).

[6] Washington Taylor IV and M. V. Raamsdonk, hep-th/9904095.

[7] B. de Wit, K. Peeters and J. Plefka, Nucl. Phys. B532, 99 (1998).

[8] E. Bergshoeff, E. Sezgin and P. K. Townsend, Phys. Lett. 189B, 75 (1987) ; Ann. Phys. 185, 330 (1988).

[9] L. Brink and P. Howe, Phys. Lett. 91B, 384 (1980) ;

E. Cremmer and S. Ferrara, Phys. Lett. 91B, 61 (1980).

[10] B. de Wit, J. Hoppe and H. Nicolai, Nucl. Phys. B305[FS23], 545 (1988).

[11] B. de Wit, M. Luscher and H. Nicolai, Nucl. Phys. B320, 135 (1989).

[12] P. K. Townsend, Phys. Lett. B373, 68 (1996).

[13] M.B.Green, M.Gutperle and H.Kwon, JHEP 9908:012 (1999) ;

S.Hyun, hep-th/9909119.

[14] E. Cremmer, B. Julia and J. Sherk, Phys. Lett. 76B, 409 (1978).

[15] S. Deser and B. Zumino, Phys. Lett. 62B, 335 (1976).

[16] A. H. Chamseddine and P. C. West, Nucl. Phys. B129, 39 (1977).

[17] R. Arnowitt and P. Nath, Phys. Lett. 56B, 177 (1975);

J. Wess and B. Zumino, Phys. Lett. 66B, 361 (1977) ; Phys. Lett. 79B, 394 (1978) ;

L. Brink, M. Gell-Mann, P. Ramondand and J. H. Schwarz, Phys. Lett. 74B, 336 (1978) ;

P. van Nieuwenhuizen and S. Ferrara, Ann. Phys. 127, 274 (1980). 
[18] A. Dasgupta, H. Nicolai and J. Plefka, hep-th/0003280. 